Bijlage IV.

\title{
RÉPARTITION DES HYPOTHÈQUES DE LA BANQUE DU BRÉSIL 3874.
}

\section{LOI No. 2400 DU 17 SEPTEMBRE 1873}

Autorise un nouvel accord avec la Banque du Brésil et réduit le rachat annuel des notes de cette Banque et des autres Banques de circulation.

Dom Pedro II, par la Grâce de Dieu et l'unanime acclamation des peuples, Empereur Constitutionel et Défenseur Perpétuel du Brésil;

Faisons savoir à tous nos sujets que l'Assemblée Générale a décretée et que Nous voulons que soit exécutée la Loi suivante:

Art. ler. La proportion marquée dans le $\S 3^{\mathrm{a}}$ de l'article ler de la Loi du 22 Août 1860 , pour le rachat des billets des Banques de circulation, est réduite à $2 \frac{1}{2} \mathrm{pCt}$. par an, cette disposition étant applicable à la Banque du Brésil, de conformité aux dispositions de cette Loi.

Art. 2ème. Le Gouvernement est autorisé à proroger pour quatorze ans de plus la durée de l'existence de la Banque du Brésil, celle-ci étant obligée à employer le capital de son portefeuille hypothécaire, qui ne sera pas inférieure à $25.000,000 \$ 000$, à des prêts faits à l'agriculture, et effectués dans les termes de cette Loi, cela dans le délai qui lui sera marqué par le même Gouvernement, et à étendre le cercle de ces transactions hypothécaires an delà de ce qui est désignée dans les statuts actuels.

Ce délai expiré, le rachat annuel des billets de la Banque sera élevé à 8 pCt. sur la différence entre le capital de $25,000,000 \$ 000$ et la somme employée effectivement sur les prêts à l'agriculture.

$\S$ ler. Déduisant la valeur représenté par des titres en liquidation dans sa section des hypothèques, la Banque du Brésil 
complètera le capital qui lui est fixé par cette Loi, comme fonds exclusif de la dite section, séparant de son portefeuille commercial pour l'hypothécaire une somme égale en apolices de la dette publique, ou en monnaie courante.

Et il comblera de la même manière tout déficit qui pourrait se présenter dans le dit capital après avoir été converti en prêts hypothécaires.

§ 2e. Dans sa section hypothécaire, la Banque du Brésil ne pourra exiger, pour les prêts qu'elle fera à l'agriculture, un intérêt supérieur à 6 pCt. par an, ni une amortisation annuelle supérieure à 5 pCt., calculée sur le total de la dette primitive, les intérêts et l'amortisation seront payés par sémestres échus.

$\S 3$. Les prêts faits par la Banque du Brésil avant la date de cette Loi, sous garantie d'hypothèques d'établissements agricoles, sont sujets aux dispositions du paragraphe précedent.

$\S 4$ e. Dans le rachat de ses billets, la Banque du Brésil donnera la préférence à ceux qui resteraient des Caisses filiales de Pernambuco, Bahia, Maranhāo et Pará.

§ 5e. Au cas ou la Banque du Brésil refuserait d'accéder à quelqu'une des dispositions des paragraphes précédents, le Gouvernement fixera une quote annuelle, du rachat de ses billets au maximum de l'art. ler $\$ 6$ e de la Loi du 12e Septembre 1866.

Art. $3^{\mathrm{e}}$. Sont révoqués les dispositions contraires.

Nous ordonnerons par conséquent aux autorités auxquelles appartient la connaissance de la dite Loi, qu'elles l'exécutent et la fassent exécuter et garder telle qu'elle est exprimée. Le Sécretaire d'Etat du Département des Finances, la fera imprimer, publier et parvenir.

Donnée dans Notre Palais de Rio de Janeiro, le 17 Septembre de l'année 1873, cinquante deuxième de l'Indépendance et de l'Empire.

L'Empereur, avec signe et sceau. - Vicomte de Rio Branco.

Lettre de Loi par laquelle Votre Majesté Impériale ordonne d'exécuter le Décret de l'Assemblée Générale, qu'elle a daigné sanctionner, concédant des faveurs à la Banque du Brésil, sous de certaines conditions.

Pour que Votre Majesté Impériale apprécie. - A été rédigée par Augusto Frederico Colin. 
ACCORD ENTRE LE GOUVERNEMEN'T ET LA BANQUE DU BRÉSIL.

\section{Exposition de motifs.}

Sire. - Le gouvernement est autorisé à innover le contrat conclu, avec la Banque du Brésil, en vertu de la Loi du 12 Septembre 1866 .

La pensée de la Loi du 17 Septembre dernier est de mettre la Banque en état de venir, plus amplement en aide à l'agriculture nationale, en emplificant ses opérations hypothécaires sur les bases de la Loi du 24 Septembre 1864, qui régularise l'organisation des sociétés de crédit réel, moyennant des conditions moins onéreuses que celles des contrats actuels.

La Loi de 1866 ordonna de séparer de l'ancien portefeuille de cette établissement, 35,000,000\$000, en titres des plus propres, à être convertis en obligations hypothécaires, mais une partie de ces titres est devenue sans valeur réelle, et toute leur importance liquide n'a pas été employée effectivement à de nouveaux prêts de cette nature.

Au 30 Juin passé, date du demier bilan, la dette hypothécaire excédait peu la somme de $18,000,000 \$ 000$, tandis que sur le fond destiné à ces opérations, il y avait plus de $5,000,000 \$ 000$ disponibles, dont $45,000,000 \$ 000$ environ employés en apolices de la dette publique.

En présence de ces faits, le législateur de 1873 a fixé à $25,000,000 \$ 000$ le capital minimum de la caisse hypothécaire de la Banque, déterminant que cette somme fut complêtée au moyen d'apolices ou de monnaie courante du portefeuille commercial, en réintegrant de la même manière, chaque fois qu'elle souffrirait une diminution par suite de préjudices.

La même loi de 1866 décretée, quand les circonstances de la Banque étaient critiques et qu'il convenait surtout de pourvoir au rachat de sa circulation fiduciaire, cette loi ne fixa pas le maximum de l'intérêt ni celui de l'amortisation des prêts constitués sur hypothèques de biens ruraux.

Le décret du 22 Juillet 1867, qui donna une réglement à la section hypothécaire, établit comme maximum de l'intérêt, celui de l'Escompte des lettres commerciales, c'est-à-dire 9 pCt. en général et, comme limite de l'amortisation, 8 pCt.

La loi nouvelle, offrant à la Banque l'avantage de racheter ses billets sur une plus petite échelle, restreignant à 6 pCt. 
l'intérêt des contrats faits avec les propriétaires agricoles, et à 5 pCt. le taux de ses paiements annuels.

L'annuité qui était, en général, de 17 pCt sera de 11 pCt. Jusqu'aujourd'hui, le receuillement des billets de la Banque a été fait annuellement dans la proportion de 5 pCt. quoique le Gouvernement pût élever ce taux jusqu'à 8 pCt. La proportion concédée actuellement est de $2 \frac{1}{2}$ pCt. calculée sur la même importance de l'émission restante en 1866 , c'est-à-dire, sur $45,600,000 \$ 000$.

Comme conséquence de cette nouvelle faveur de la Loi et de l'obligation de prêter à de longs délais, la durée de la Banque a été prolongée de 14 années, son existence se terminant sans cela en 1886 .

D'un côté, comme il serait périlleux de forcer l'emploi de $25,000,000 \$ 000$ dans un délai très court et fatal, ou les garanties dont les prêts hypothécaires doivent être revêtus et la lenteur, inévitable qui en résulte, d'autre part, comme la loi doit assurer son exécution fidèle en ce qui touche la somme des avantages promis à l'industrie agricole, le législateur a adopté une décision raisonnable entre la rigueur excessive et la confiance absolue.

Cette résolution consiste en l'élévation du taux du rachat des billets de la Banque, en relation à la somine qui manquera, pour que les prêts hypothécaires faits à l'agriculture parfassent le minimum de $25,000,000 \$ 000$ le rachat se fera, non dans la proportion de $2 \frac{1}{2}$ pCt., mais dans celle de 8 pCt. sur le chiffre égal à la différence entre ce capital et l'importance de ces contrats.

De cette manière il $\mathbf{y}$ aura une instigation à ce que la Banque, loin de restreindre ses secours à l'agriculture, les amplifie, au moins dans ces limites.

Si la Banque n'annuait pas à ces conditions, la quote de l'extinction annuelle de ses billets devrait être fixée à 8 pCt., maximum autorisé par la loi du $] 22$ Septembre 1866.

Les conditions de la Loi du 17 Septembre ont été acceptées, et après avoir entendu la commission des Finances du Conseil d'Etat, sur le projet du nouveau contrat proposé par le président de cet établissement, ainsi que sur les amendements, qui me paraitront nécessaires, et les observations que ceux-ci susciteront de la part de la même Banque, il a été formulé enfin l'accord que j'ai maintenant l'honneur de soumettre à l'examen et à l'approbation de V. M. Impériale. 
Toutes les dispositions de la Loi y sont scrupuleusement respectées.

Non seulement on a prêté une attention toute particulière au précepte spécial du législateur dans la fixation du capital destiné aux prêts sur biens ruraux, mais l'on a aussi cherché à augmenter les ressources de la caisse hypothécaire par une dérogation à l'art. 8 des statuts de 1870 .

La Loi et les statuts de 1866 ont marqué pour limite du fond de réserve de la Banque de 30 pCt. de tout son capital réalisé; mais la réforme de 1870 éleva cette garantie à $50 \mathrm{pCt}$.

Aujourd'hui l'on peut en revenir au maximum de la Loi du 12 Septembre 1866 et renforcer avec les 20 pCt. restants le capital des opérations hypothécaires.

Il est vrai que le fonds de réserve étant obligé de couvrir les préjudices resultant des transactions annuelles de la Banque, il peut descendre de ce maximum après l'avoir atteint, et que, puisqu'il est nécessaire de le conserver intégralement, il n'y a pas, par conséquent l'augmentation désirée du capital de la caisse hypothécaire; mais les effets de, cette eventualité sont atténués par la restriction du quantum des dividendes, qui ne peut excéder de 10 pCt. selon le même art. 8 des statuts de 1870 , restriction qui n'a pas cessé d'exister.

La sanction de la loi pour atteindre l'emploi effectif de 25,000,000\$000 en faveur de l'agriculture a été appliquée de la manière la plus efficace, en conciliant la prudence requise par la Banque qui craignait de se compromettre par un délai inférieur à trois années, et l'intérêt public qui doit correspondre à la faveur importante concédée maintenant à cette établissement.

La Banque aura une année pour remplir cette indéclinable disposition de la loi, jouissant de la pleine faveur du rachat de ses billets, en raison de $2 \frac{1}{2}$ pCt., si cependant, à la fin de l'année, elle n'a pas réalisé les $25,000,000 \$ 000$ de prêts hypothécaires, le taux du rachat sur la différence sera de 4 pCt. l'année suivante, de 6 pCt. pour la troisième année et de 8 pCt. à partir de la 4 me .

La circonscription territoriale pour les opérations hypothécaires est considérablement élargie.

Aujourd'hui elle ne comprend que le municipe de la capitale de la province de Rio de Janeiro et les municipes limitrophes de S. Paulo, Minas Geraes et Espirito Santo; par l'accord elle 4e Volgr. IX. 
s'étend à tous les municipes de ces provinces et à celles du Paraná et de Santa-Cathérina.

Le gouvernement ne refuserait pas une plus grande circonscription, mais l'administration de la Banque ne l'a pas jugé praticable en alléguant des considérations très serieuses et elle a demandé à être dispensée d'une faculté qui pourrait lui amener des plaintes d'espérances trompées, et rendant peut-être difficile l'organisation d'autres sociétés locales.

La nouvelle Loi a étendu ses bienveillantes dispositions aux prêts réalisés antérieurement sur hypothèques d'établissements agricoles.

Cette mesure salutaire est consacrée dans le paragraphe unique de l'article $7_{\mathrm{e}}$ du présent accord, permettant que, même les débiteurs qui se seraient mis en défaut de ponctualité pussent profiter des nouvelles conditions sous la forme qu'ils pourraient décider avec le créancier comme l'avait prévu la législation en vigueur, ou les conditions plus favorables.

La section hypothécaire de la Banque du Brésil étant constituée en parfaite institution de crédit réel, conformément aux principes de la loi du 24 Septembre 1864, l'occasion était arrivée d'user de la faculté qui lni était garantie par l'article 7 e des statuts de 1866 , celle d'émettre des lettres hypothécaires.

En effet, la Banque se propose d'entrer dans cette ordre d'opérations de crédit, au moyen desquels elle pourra faire des prêts jusqu'au décuple du capital de la caisse hypothécaire, une fois qu'elle aura réussi à attirer une partie des capitaux disponibles pour ces titres de petite valeur, suffisamment garantis, qui donnent un intérêt et sont transférables comme ceux du trésor pouvant avoir en outre la perspective d'un prix dans leur amortisation annuelle par voie du sort.

L'émission des lettres hypothécaires commence à peine à être essayé entre nous par la Banque Prédial de cette Capitale, et il n'y a pas une association brésilienne qui soit en des conditions aussi avantageuses que la Banque du Brésil pour accréditer cet instrument économique en le nationalisant, pour ainsi dire, dans l'Empire, dont l'agriculture le sollicite et récompensera abondamment ses services.

La Loi du 24. Septembre 1864 autorise l'emploi des fonds des sociétés de Crédit réel et même ses dépôts en apolices de la dette de l'Etat et en billets du trésor. 
La section hypothécaire de la Banque, se plaçant sous le régime de cette loi, la permission qu'elle avait par ses statuts d'employer en apolices le fonds disponible de cette caisse, est extensive non seulement aux billets du trésor, mais aussi aux lettres hypothécaires elles-mêmes qui selon la loi précitée de 1864, peuvent dispenser les emprunts en numéraire selon la volonté des emprunteurs.

Cet emploi des lettres hypothécaires sera un moyen de plus de profiter leur crédit et d'animer leur circulation.

Suivant en cela l'exemple de la Société du Crédit-Foncier de France, l'administration de la Banque a jugé que, pour motif d'ordre et de régularité dans les écritures, ses lettres hypothécaires ne doivent donner des intérêts qu'au semestre suivant celui de l'émission.

En outre qu'il était autorisé par la susdite association, ee système a paru sans inconvénient à la commission des Finances du Conseil d'Etat, sauf ce que notre propre expérience pourrait nous enseigner à ce sujet.

Il n'y a aucun préjudice pour l'une ou l'autre des parties intéressées dans la transaction, ni pour ceux qui reçoivent ces titres de la section quand elle prête dans la même espèce

Dans le premier cas, l'établissement paie à qui lui achète la lettre l'intérêt du temps qui manque pour compléter le semestre de l'émission, et, ainsi, il n'y aura pas d'intérêt à payer avant le semestre suivant; dans le second cas, l'opéraration a lieu en sens invers: l'emprunteur qui reçoit la lettre comme prêt, paie à la section hypothécaire l'intérêt compté depuis ce jour jusqu'à la fin du semestre en lequel est fait le contrat.

Le décret et le règlement du 22 juillet 1867 défendent des prêts inférieurs à $10,000 \$ 000$ ou supérieurs à $120,000 \$ 000$.

Ce minimum avait sa raison d'être dans la garantie précaire d'établissements agricoles de si petite importance.

Et cependant, puisque cette restriction a été censurée dans le Sénat comme excessivement rigoureuse pour la petite agriculture, on a jugé convenable de la supprimer, maintenant que l'on dote mieux la section hypothécaire et que la Banque inspire assez de confiance par sa solidité et par l'expérience de son administration.

Le maximum de $120,000 \mathrm{\$} 000$ a été maintenu pour que l'aide de la Banque soit la plus étendue et la plus équitable 
possible, et ce maximum ne sera certainement pas considéré comme très restraint si l'on a égard à la règle légale qui défend des emprunts excédant la moitié ou les trois quarts de la valeur des biens hypothéqués, selon qu'ils sont ruraux ou urbains.

La nature et l'étendue des nouvelles opérations exigent non seulement la complète séparation des capitaux et des responsabilités des deux caisses, la commerciale et l'hypothécaire, mais aussi que les dispositions de l'article 13 , $\$ \$ 14$ et 15 de la loi de 1804, qui exceptent de la faillite commerciale les sociétés de crédit réel, s'appliquent aussi à la section des hypothèques.

Telles sont, Sire, les altérations faites par l'accord, auquel je me réfère, aux statuts actuels de la Banque du Brésil.

Elles recevront immédiatement leur développement naturel logique et ont été rédigées avec la connexion nécessaire avee les autres articles des mêmes statuts, en lesquels rien n'a été innové, et qui ont été à peine modifiés en leur forme.

J'ai l'honneur d'être, Sire, avec le plus profond respect, de V. M. Impériale, le très révérencieux serviteur,

Rio de Janeiro,

24. Décembre 1873.

Vicomete de Rio Branco.

DECRET $\mathrm{N}^{\circ} .5506$ DU 26 DÉCEMBRE 1873

Approuve et confirme laccord du 24, courant, intervenu entre le Ministre des Finances et la Banque du Brésil.

Je daigne approuver et confirmer l'accord du 24, courant, intervenu entre le Ministre des Finances et le Président et les membres du conseil, directeurs de la Banque du Brésil, pour l'exécution de la Loi No. 2400 du 17 septembre 1873, qui suit le présent decret.

Le Vicomte de Rio Branco, conseiller d'Etat, Sénateur de l'Empire, Président du Conseil des Ministres, Ministre et Sécretaire d'Etat au Département des Finances et Président du Tribunal du Trésor national, le comprenne ainsi et le fasse exécuter.

Palais de Rio de Janeiro, 26 décembre de l'année 1873, cinquante deuxième de l'Indépendance et de l'Empire. - Avec la signature de l'Empereur. - Vicom'te de Rio Branco. 
Accord effectué entre le Gouvernement et la Banque du Brésil pour l'exécution de la loi No. 2400 du 17 Septembre 1873.

Le vingt-quatrième jour du nois de décembre de l'année 1873 , dans cette capitale et très loyale et héroique ville de S. Sébastien de Rio de Janeiro, et dans la salle du Tribunal du Trésor national, entre l'Tllme et Exme Sieur Vicomte de Rio Branco, Ministre et Sécretaire d'Etat au Département des Finances et les Sieurs José Machado Coelho de Castro, Vicomte de Tocantins, Dr José Fernandes Moreira, Conseiller Joāo Manuel Pereira da Silva, Commandeurs Joāo Baptista do Fonseca et Antonio José dos Santos, Président et Membres du Conseil directeur de la Banque du Brésil, pleinement et dûment autorisés, a été décidé que le contrat du 11 octobre 1866, conclu entre le Gouvernement et la Banque est renové pour l'exécution de la Loi No. 2400 du 17 septembre 1873, dans la forme exprimée par les articles qui suivent.

Art. ler. La Banque du Brésil, dont la durée d'existence est prolongée jusqu'au 31 décembre 1900 , continuera a être divisée en deux sections distinctes, quoique sous la même administration: la commerciale et l'hypothécaire (Iois No. 1349, art. ler $\S 1$ er et $\mathrm{N}^{\circ} 24,00$ art. $\left.2 \mathrm{e}\right)$.

Art. 2e. Le fonds de la caisse hypothécaire, destinée spécialement à des prêts sur biens ruraux, ne sera pas inférieure à $25,000,000 \$ 000$ et devra être immédiatement complétée en monnaie courante ou en apolices de la dette publique, retirées du partefeuille commercial et ne comptant pas pour cette computation les titres actuels en liquidation de la section hypothécaire (Loi No 2400 , art. $2 \mathrm{e} \$ 1^{\mathrm{er}}$ ).

$\S 1^{\mathrm{er}}$. Le fonds de réserve dont il est question dans l'art: 8 me des statuts approuvés par le décret $\mathrm{N}_{\mathrm{o}} .4556$ du 10 août 1870 est limité à 30 pCt. du capital réalisé de la Banque, et le fonds spécial de la caisse hypothécaire s'augmente des 20 pCt. restants.

Chaque fois que, pour des motifs vérifiés dans le recouvrement de titres en liquidation, le fonds de réserve descendra de ce maximum, ou suspendra la dite augmentation du fonds spécial de la caisse hypothécaire jusqu'à réintrégrer les $30 \mathrm{pCt}$, en observant en tous cas, ce que dispose le précité art. 8 des statuts de 1870 quant au dividende le plus élevé qui puisse être distribué annuellement aux actionnaires. 
$\S 2$ me. Aucune partie du fonds d'une des deux caisses, commerciale et hypothécaire, ne pourra être appliquée à des opérations de l'autre, excepté dans les cas où il s'agira de faire ce qui est prescrit dans le présent article (Lois No. 1349, art. ler $\S 1 \mathrm{er}$ et No. 2400 art. 2e.)

$\S 3$ e. Après avoir converti en prêts le fonds destiné à la section hypothécaire, tout déficit, que souffrirait cette caisse, serait comblé de la manière déterminée dans la première partie de l'art. 2 .

$\S$ 4. Le fonds disponible de la caisse hypothécaire qui ne pourra être employé immédiatement en prêts à l'agriculture, pourra l'être provisoirement en apolices de la dette publique en billets du Trésor national et en lettres hypothécaires de la Banque même ou d'un autre établissement de crédit foncier. (Lois No. 1349 art. 18 et No. 1237 de 1864 art. $13 \$ 16$. )

Art. 3e. La proportion marquée dans l'art. ler $\$ 6$ de la loi $\mathrm{N}^{\circ}$. 1349 du 12 septembre 1866 pour le rachat des billets de Banque qui sont encore en circulation est réduite à $2 \frac{1}{2} \mathrm{pCt}$. annuellement de son importance primitive; et ne pourra être altérée jusqu'à la fin du délai de la durée de l'établissement désigné dans l'art. $1^{\mathrm{er}}$; la dite circulation devant être éteinte dans ce délai (Lois No. 2400 art. 1 ${ }^{\mathrm{er}}$ ).

$\mathrm{Si}$ cependant au bout de la première année à compter du ler Janvier 1874, la Banque n'avait pas employé effectivement en prêts à l'agriculture et sous les conditions de la loi No. 24,00 du 17 septembre 1873, au moins la somme de 25,000,000\$000, la proportion de $2 \frac{1}{2}$ pCt. pour le rachat de ses billets, elle sera élevée à 4 pCt. sur la différence entre cette somme et la proportion des dites prêts.

La seconde année terminée, la dite proportion sera de 6 pCt. et, à partir de la quatrième année, de 8 pCt. calculée sur la différence indiquée.

$\S 1$ er. Dans le rachat de ses billets, la Banque du Brésil donnera préférence à ceux qui resteraient des caisses filiales de Bahia, Pernambuco, Maranhāo et Pará. (Loi précité No. 2400 art. $\left.2 \theta \S 44^{e}\right)$.

Art. 4e. Les opérations de la section hypothécaire consisteront:

10. En prêts sur hypothèques d'immeubles ruraux ou urbains à longs délai, de 10 à 25 ans, payables par annuités successives.

2o. En prêts sur hypothèques d'immeubles ruraux ou urbains à court délai, de moins de 10 ans, avec ou sans amortisation. 
$3^{\circ}$. En l'émission et la négociation de lettres hypothécaires fondées sur les prêts à long délai, dans la forme de l'art. 13 de la loi du 24 septembre 1864. (Décret du 10 août 1870 , art. 59 , et du 22 juillet 1867 , art. 2 e).

Art. 5e. Dans les emprunts, tant à long délai qu'à court délai, l'on observera les règles contenus dans les paragraphes suivants :

\$ ]er. La circonscription territorial de ces opérations comprendra le municipe de la Capitale et les provinces de Rio de Janeiro, Espirito Santo, Minas-Geraes, Sāo Paulo, Pará et Santa Catherina, sauf la faculté d'admettre, par exception, des hypothèques sur des immeubles situés sur d'autres points de l'empire dans le but de renforcer les garanties des titres de dette existant dans les portefeuilles de la Banque. (Loi No. 2400 de 1873 art. 2e. Décret du 22 juillet 1867, art. 1er; Décret du 3 juin 1865, arts. 2e et $3 \mathrm{e})$.

$\$ 2 \mathrm{e}$. Thes prêts ne pourront être faits que sur première hypothèque, constituée, cédée ou subrogée, de conformité à la loi $\mathbf{N}_{\text {o. }} 1236$ de 1864 et les règlements expédiés pour son exécution. (Décret No. 3471 de 1865, art. 18).

$\S 3$ e. T.es emprunts destinés au paiement d'hypothèques inscrites antérieurement n'auront lieu que lorsque par ce paiement, l'hypothèque cédée prendra la première place et sans concurrence; et tant qu'il restera au pouvoir de la section hypothécaire la somme nécessaire au paiement du principal de la dette, des intérêts échus et à échoir jusqu'à l'époque d: paiement et la somme nécessaire aux dépenses de la subrogation (Décret de 1865, art. 19).

Ainsi restera également retenu au pouvoir de la-dite section. la somme nécessaire pour payer le principal et les intérêts des dettes, dont la garantie hypothécaire devra être distribuée entre les créanciers respectifs, ou que les mêmes immeubles resteront hypothéqués par elle.

$\S 4^{\mathrm{e}}$. Le délai des prêts ne pourra excéder, en aucun cas : celui de la durée assignée à la Banque par l'article premier de ce règlement.

$\S 5^{\mathrm{e}}$. Aucun prêt n'excédera la moitié de la valeur des immeubles ruraux, et les trois quarts de celle des immeubles urbains (Loi de 1865 , art. $13 \mathrm{e}, \$ 5$ ).

$\$ 6^{\mathrm{e}}$. Aucun prêt ne pourra dépasser la somme de $120,000 \$ 000$ (1)écret de 1867, art. 3e). 
$\S 7$. Seront exclues de l'hypothèque pour les prêts hypothécaires, les propriétés de rendement précaire et celles de valeur vénale de réalisation difficile. (Décrets de 1865, art. 7, $\S 5$ et de 1867 , art. 4).

$\S 8$ e. Les propositions ou demandes d'emprunt contiendront la désignation des immeubles et leurs rendements, avec un évaluation particulière de chaque article, et seront accompagnés de tous les documents et toutes les informations qui justifient le droit d'hypothèques dans la forme de la législation en vigueur.

Le contrat ne sera conclu qu'après que l'évaluation aît été vérifiée par le procédé désigné dans l'art. 58 des statuts, approuvés par le décret №. 4566 de 1870 et qu'on aît rempli les formalités prescrites dans le réglenient intérieur de la section hypothécaire. (Décret de 1867, art. 5e).

$\S 99^{\circ}$. Toutes les dépenses effectuées par la section hypothécaire pour les examens et les évaluations des immeubles seront faites pour le compte de ceux qui auront requis l'emprunt et même au cas ou celui-ci n'aurait pas lieu. (Décret et art. cité $\S \mathrm{ler})$.

$\S 10$ e. La faute du paiement, à l'époque déterminée de la prestation stipulée dans le contrat que ce soit paiement d'intérérêts ou d'amortisation du capital, donnera à la section hypothécaire le droit de recouvrer, pour le retard l'intérêt qui sera conventionné et également celui de réclamer le remboursement de la totalité de la dette. (Loi du 24 septembre 1864, art. 4 e $\S 9$ et décret de 1867 art 8 e).

Art. 6e. Dans les contrats qu'elle fera, la section hypothécaire pourra imposer les conditions suivantes :

10 Devenir exigible toute la dette et l'emprunteur soumis à payer un indemnisation de 5 pCt. de son importance, si dans le délai d'un mois, il ne dénonce pas l'aliénation totale ou partielle qu'il aura fait de l'immeuble hypothéqué, les détériorations que celui-ci aura souffertes, et les évènements qui en diminuent la valeur et en perturbent la possession de même que si le dit emprunteur cache des faits à lui connus qui produisent la dépréciation de l'immeuble et annullent ou rendent douteux son droit de propriété. (Décrets de 1865, art. 7e et de 1867 art. 9e.)

2o. L'emprunteur s'oblige à assurer la partie édifiée de la propriété contre les risques d'incendie , chaque fois que ce sera 
possible, et l'assureur devant être indiqué par la section hypothécaire, l'assurance étant maintenue pendant tout le temps -de l'existence de l'emprunt.

La section hypothécaire pourra aussi renouveler l'assurance en payant la prime, celle-ci étant portée au compte de l'emprunteur (Décret de 1867 art. 6e).

Art. 7e. Dans les prêts hypothécaires aux agriculteurs, il ne pourra être exigé un intérêt supérieur à $6 \mathrm{pCt}$. par an, ni d'amortisation annuelle qui dépasse 5 pCt. de l'importance primitive de la dette.

Les prestations d'intérêt et d'amortisation serout payées par semestres échus.

§ Unique. Les prêts réalisés par la Banque jusqu’à la dâte du présent règlement sous garantie d'hypothèque d'établissements agricoles, sont sujets à la disposition de cet article, en augmentant le délai dans les conditions qui seront fixées d'accord avec les emprunteurs, et cela aussitôt que les dits emprunteurs se prèteront à l'accord nécessaire sur le mode de satisfaire, l'obligation imposée par le paragraphe 10 de l'art. 5.

Art. 8e. Dans les opérations et contrats à longue échéance, on observera, en outre des préceptes consignés dans les articles antérieurs, les règles spéciales suivantes:

$1^{\circ}$. Les prêts hypothécaires seront faits en argent ou en lettres hypothécaires au pair, au choix de l'emprunteur. (Ioi de 1864, art. 13, § 11. Décret de J865, arts 21, 22 et 23.).

$2^{\circ}$. Les prêts hypothécaires seront payables par annuitées successives ou par anticipations (Loi de 1864, art. 13, $\$ \$ 7$ et 9).

3o. Les annuitées seront calculées de sorte que l'amortisation totale soit réalisée en 10 ans au moins, et en 25 au plus. (Loi de 1864 art. $13 \S 7$ ); elles comprendront l'intérêt stipulé, la quote d'amortisation et pour-cent de l'administration qui n'excédera pas 2 pCt. pour les prêts ruraux et 4 pCt. pour les urbains (Art. 13, précité $\$ \S 8$ et 9 ) et seront payées en argent par semestres échus.

4o. Les paiements par anticipation pourront être de toute la dette ou partielle, se réduisant dans le second cas, en proportion des annuitées effectuées en argent ou en lettres hypothécaire le droit de recouvrer dans le même acte une indemnisation qui n'excédera jamais $\mathbf{l}$ pCt. de la somme remboursée effectivement. (Loi de 1864, art. 13, $\$$ 9. Décret de 1867 arts. 34 et 38$)$. 
$5^{\circ}$. L'enchère ou l'adjudication des immeubles pour payements de la section hypothécaire, dans les prêts à longs délais, sera exemptée de l'impôt de transmission. (Loi 1864, art. 13, §12).

Art. 9e. L'émission des lettres hypothécaires ne pourra excéder la somme de la valeur nominale des emprunts à longs délais, ni le décuple du capital de la section hypothécaire; de même que le total de la valeur nominale de celles qui seraient en circulation n'excédera pas la somme dont l'établissement sera créancier pour ces prêts. (Loi de 1864, art. 13, § 6. Décret de 1865 art. 40 et 49 .)

$\S \mathrm{Ir}^{\mathrm{r}}$. Des lettres hypothécaires auront leurs numérations d'ordre qui sera relative à l'année - de leur émission; elles seront extraites d'un livre spécial à touche et signées par un membre du Conseil directeur et par le Président de la Banque. (Décret de 1865 . art. 48$)$.

$\S 2$. Elles seront nominatives et comme telles transférables par endos, mais seulement avec effet de cession civile, ou au porteur et transférables par simple remise (Loi de 1864, art. 12, $\$ \$ 2$ et 3. Décret de 1865 , art. 41 et 44 ).

\$ 3. Les lettres hypothécaires et leur transfert seront excemptés du timbre proportionnel. (Art. 13, § 12, de la Loi de 1864).

\$ 4. Elles devront spécifier le capital qui ne sera jamais inférieur à $100 \$ 000$ (Loi de 1864 , art. $13, \$ 4$ ), et leur taux d'intérêts, leur temps et leur mode de paiement, qui aura lieu par semestres échus. (Décret 1855, art. 55 et 56).

§ 5. Si l'émission des lettres, en outre des conditions spécifiées ci-dessus offrent celle de prix par tirage au sort, leur importance et leur distribution seront marquées par le Conseil directeur et devront être mentionnées dans les lettres.

§6. La date du paiement de l'intérêt des lettres devra être combinée avec celle des paiements des anmuitées, de manière à ce qu'il existe une moyenne de trois mois au moins, pendant lesquels la section hypothécaire puisse recouvrer de ses débiteurs les annuités avec lesquelles elle doit payer les intérêts. (Loi de 1864, art. 13, §9. Décret de 1865, art. 57).

$\S 7$. Les lettres hypothécaires n'auront pas d'époque fixe pour leur paiement, mais elles seront payées par voie du tirage au sort, qui aura lieu au jour désigné par le Conseil directeur, une fois par an et en présence d'un membre du dit conseil, par la manière et dans les buts désignés dans les articles 51 , 52,54 et 61 du règlement du 3 juin 1865 . 
\$ 8. On lèvera un acte dans uu livre spécial de toute la procédure du tirage au sort, et de l'annullation des lettres hypothécaires et une copie en sera envoyée au Ministre des Finances.

$\$ 9$. Les lettres émises durant le sémestre ne donneront droit qu'aux intérêts du semestre suivant; mais les porteurs ou souscripteurs payeront en moins la somme équivalante aux intérêts comptés du jour de l'émission jusqu'à l'échange du premier coupon semestriel, qui sera détaché de la lettre.

De conformité avec cette disposition, la section hypothécaire, dans ses prêts, recevra immédiatement de l'emprunteur ou déduira du capital que celui-ci devra recevoir, l'intérêt correspondant aux mois on jours qui courront, depuis la dâte du contrat jusqu'à la fin du semestre en lequel le dit contrat se fera. (Art. 24 du Décret Ne. 3471 du 3 juin 1865).

Les porteurs des lettres hypothécaires pourront les disposer à la Banque, recevant un certificat qui servira de titre pour le recouvrement des intérêts.

Pour ce service, la section hypothécaire percevra la commission de $\frac{1}{8} \mathrm{pCt}$.

Art. 10e. Les porteurs des lettres hypothécaires ne pourront avoir d'action que contre la section hypothécaire. (Loi de 1864; art. $13, \S 13)$.

Art. 1」e. Ta Section hypothécaire pourra acquérir de ses débiteurs par des moyens conciliatoires, les biens qui lui seront hypothéqués.

Paragraphe unique. De la même forme, elle pourra acquérir les dits moyens judiciaires, dans les cas suivants:

$1^{\circ}$. Par la voie d'adjudication dans les exécutions d'actes hypothécaires qui sont de sa compétance en vertu de la loi du 24 septembre 1864 et du réglement No. 3453 du 26 avril 1865.

1. Par voie de licitation dans les cas de rémission requise par l'acquéreur de l'immeuble hypothéqué dans les termes des articles 299 et 300 du réglement No. 3453 de 1865.

Art. 12. L'acquisition par moyens conciliatoires ou l'exécution judiciaire n'étant pas de la convenance de la section hypothécaire elle pourra requérir le sêquestre des immeubles hypothéqués pour se payer par leurs revenus aux choix d'un des moyens suivants:

§ ler. En convertissant le séquestre en dépôt au pouvoir du débiteur, celui-ci acceptant l'obligation comme dépositaire judi- 
ciaire, de remettre les fruits et rendements sauf déduction des dépenses convenues entre lui et la section hypothécaire.

§ 2e. En convertissant le sequestre en antichrèse, la section hypothécaire requérant l'émission dans la possession des biens pour les administrés jusqu'au paiement des annuitées, des intérêts et des dépenses de l'administration. (Décret Nó. 3417, de 1865 art $^{\mathrm{s}} 70$ et 71$)$.

Art. 13e. La section hypothécaire de la Banque n'est pas sujette à la faillite commerciale lui étant applicables les dispositions de la loi no. 1327 du 24 septembre 1864 art. 13, $\S \S 14$ en 15.

En témoignage de quoi et pour qu'il soit constant a été dressé le présent accord en double, signé par les susdits Exme Sieur Vicomte de Rio Branco, Ministre des Finances et Sieurs José Machado Coelho de Castro, Vicomte de Tocantins, Dr. José Fernandes Moreira, Conseiller Joāo Manuel Pereira da Silva, les Commandeurs Joāo Baptista do Fonseca et Antonio José dos Santos, Président et membres du Conseil directeur de la Banque du Brésil, avec le sceau des armes de l'Empire.

Et moi José Séveriano da Rocha, officier supérieur du Sécretariat d'Etat du Département des Finances, l'ai soussigné. 1

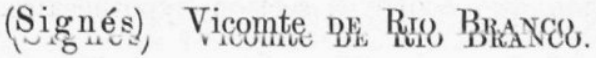 \\ José Machado Coelho de Castro. \\ Vicomte DE Tocantins. \\ José Fernandes Moreira. \\ Joāo Manuel Pereira da Silva. \\ Antonio José dos SAntos. \\ Joāo Baptista do Fonseca.
}

1 Uit het portugeesch vertaald door een beëedigd translateur te Rio. 